\title{
Explizite Lösungen einer Klàsse nichtlinearer partieller Differentialgleichungen
}

\author{
H. HEROLD
}

Für eine die.Gleichung von Boussinesq umfassende Klasse nichtlinearer partiellér Differentialgleichungen wird die vollständige Lösung eines Anfangswertproblems in expliziter Form mittels 'der Weierstraßschen' $p$.Funktion angegeben.

Д-' некоторого класса нелинейных пифффереициальных уравнений в частных производных, вюлючающего уравнение_Бусснке, дается полное решение задачи Коши в явнол форме посредством $p$-функции Веперштрасса:

For a class of nonlinear partial differential equations comprising the equation of Boussinesq the complete solution of an initial value problem is, given in explicit form by means of the Weierstrass $p$-function.

Unter den in der mathematischen Physik vorkommenden nichtlinearen partiellén Differentialgleichungen für eine reellwertige Funktion $v \doteq v(x ; t)(x, t \in \mathbb{R})$, in denen die unabhängigen Variablen nicht explizit vorkommen, sind neben der (nicht́linearen) Schrödinger-Gleichung und der,Gleichung, von Korteweg-de Vries von Wichtigkeit Gleichungen der Form

$$
\left(\frac{\partial^{2} v}{\partial t^{2}}-\frac{\partial^{2} v}{\partial x^{2}}\right) \dot{a}+\frac{\partial^{2} v}{\partial x^{2}}-6 v^{2}-c=0 \quad(a, c \in \mathbb{R}, c \neq 0)
$$

sowie

$$
\left(\frac{\partial^{2} v}{\partial t^{2}}-\frac{\partial^{2} v}{\partial x^{2}}\right) a+\frac{\partial^{i+1}}{\partial t^{i} \partial x^{j}}\left(\frac{\partial^{2} v}{\partial x^{2}}-6 v^{2}\right)=0 \quad\left(i, j \in \mathbb{N}_{0}\right)
$$
$((i, j)+(0,0))$, wobei der Spezialfall $\frac{\partial^{2} v}{\partial t^{2}}-\frac{\partial^{2} v}{\partial x^{2}}+\frac{\partial^{2}}{\partial x^{2}}\left(\frac{\partial^{2} v}{\partial x^{2}}-6 v^{2}\right)=0$ Glèchung
von Bóussinesq, heiBt.

Bemerkung: Jede Differentialgleichung der Form

$$
\left(\frac{\partial^{2} v}{\partial t^{2}}-\frac{\partial^{2} v}{\partial x^{2}}\right) c_{1}+\frac{\partial^{2} v}{\partial x^{2}}-c_{2} v^{2}-c_{3} v-c_{4}=0 \quad\left(c_{j} \in \mathbb{R}, c_{2} \neq 0\right)
$$

érhält durch die Transformation $v \rightarrow c_{2}^{-1}\left(6 v^{\prime}-c_{3} / 2\right)$ die Gestalt (1).

-.Gleichungen dieses Typs treten auf bei der Behandlung voń Wärmeleitungs- und Elastizitätsproblemen fester Materie sowie bei-der Untersuchung hydrodynamischer iVorgänge in kompressiblen Flüssigkeiten.

Bei der Untersuchung nichtlinearer partieller Differentialgleichungèn stehen allgemeinere Lösúngsmethoden zumeist 'nicht zur Verfügung, odèr allgemeinere'Lösungsansätze erfordern einen kaum zu bewältigenden mathematischen Aufwand. Reihenansätze führen beispielsweisè durchwegs auf recht verwickelte Rekursions- 
beziehungen. So sind bei der Behandlung nichtlinearer partieller Differentialgleichungen 'der Physik vielfach spezielle, von dèr jewëligen physikalischen Problemstellung ab̆hängige Lösungsverfahren erforderlich und angemessen, um einen befriedigenden Einblick in den physikalischen Charakter der Lösungen zu erhalten.

Die Gleichungen (1), (2) besitzen Lösungen der Form $v=v(\xi)$ mit $\xi=x-t$ (bzw. $\xi=x+t$ ): Offenbar ist $v$ genau dann eine Iösung vón (1), wenn $v$ eine Lösung -der Differentialgleichung

$$
d^{2} v / d \xi^{2}=6 v^{2}+c
$$

ist. Jede dẹrartige Lösung von (1) ist dann auch eine Lösung von (2). Im folgenden werden (abgesëhen von einem elementaren Fall) sämtliche lösungen vion (1) der Form $v=v(\xi)$ explizit mittels der Weierstraßschen $p$-Funktion angegeben. Aufgrund der zur Verfügung stehenden umfassenden Theorie der. $p$-Funktion wird hierdurch eine eingehende, für die jeweilige Fragestellung zweckmäßige. Untersuchung der Lösungen ermöglicht. Eine in der Umgebung von $\left(x_{0}, t_{0}\right)$. definierte Lösung $v=v(x-t)$ von (1) ist somit eindeutig festgelegt durch $v\left(\xi_{0}\right), v^{\prime}\left(\xi_{0}\right)$ mit $\xi_{0}=x_{0}-t_{0}$. Da mit $v(x-t)$ auch $v(x-t+$, const) Lösung von (1) ist, kann man sich auf den Fall $\xi_{0}=0$ beschränken.

Sei $g_{2}:=-2 c, g_{3} \because=4 \alpha^{3}-\alpha g_{2}-\beta^{2}$ mit $\alpha, \beta \in \mathbb{R}$. Die Lösung $v=v(\xi)$ von (3) mit $v(0)=\alpha, v^{\prime}(0)=\beta, v(\xi) \neq \alpha$, ist eine Lösung der Differentialgleichung $v^{2}=4 v^{3}$ $=g_{2} v-g_{3}$, wie durch Multiplikation von (3) mit $v^{\prime}$ und anschließende Integration folgt. Der elementar integrierbare Fall $g_{2}{ }^{3}=27 g_{3}{ }^{2}$ sei stets ausgeschlossen. Mit $p(z)$ werde dann die durch $g_{2}, g_{3}$ eindeutig bestimmte und für reelles $z$ reellwertige Weierstraßsche $p$-Funktion bezeichnet, $\left(2 \omega_{1}, 2 \omega_{2}\right)$.'sei ein primitives Periodenpar. Bekanntlich gilt

$$
p^{\prime}(z)^{2}=4 p(z)^{3}-g_{2} p(z)-g_{3} \quad(z \in \mathbb{C})
$$

das Polynom $P(u):=4 u^{3}-g_{2} \dot{u}-g_{3}$ hat die (voneinander verschiedenen) Nullstellen $p\left(\omega_{1}\right), p\left(\omega_{2}\right), p\left(\omega_{1}+\omega_{2}\right)$ (eine oder alle reell). Sei $u_{0}:=p(\omega)\left(\omega \in\left\{\omega_{1}, \dot{\omega}_{2}\right.\right.$, $\left.\omega_{1}+\omega_{2}\right\}$ ) die größte reelle Nullstelle voń $P(u)$ mit $u_{0} \leqq \alpha$ (mań beachte: $P(\alpha)=\beta^{2}$ ).

Dann ist, im Falle $\beta \neq 0, P(u)>0$ für $u_{0}<u \leqq \alpha$, und es sei $\eta:=\int_{u_{0}}^{j \alpha} P(u)^{-1 / 2} d u>0$. Da gemäß (4) die Umkehrung von $w=p(z)$ durch $z=\omega+\int_{u_{0}}^{w} P(w)^{-1 / 2} d w$ gegeben ist, gilt $p(\omega+\eta)=\alpha$. Durch.Anwendung des Additions-Theorems

$$
p(z+\zeta)=\frac{1}{4}\left(\frac{p^{\prime}(z)-p^{\prime}(\zeta)}{p(z)-p(\zeta)}\right)^{2}-p(z)-p(\zeta) \text { für } \zeta=\omega
$$

folgt wegen $p^{\prime}(\omega)=0$

$$
\ddot{p}(z+\omega)=1 / 4\left(p^{\prime}(z) /\left(p(z)-u_{0}\right)\right)^{2}-\dot{p}(z)-u_{0} .
$$

,Also ist $p(z+\omega)$ für $z \in \mathbb{R}$ reellwertig und (wegen $p(z)=p(-z)$ ) gerade. Es folgt

$$
\dot{p(\omega-\eta)}=p(\omega+\eta)=\alpha, \quad p^{\prime}(\omega-\eta)=-p^{\prime}(\omega+\eta) .
$$

Unter 'Beachtung von $u_{0}<p(z+\omega)<p(\dot{\omega}+\eta)$ für $0<z<\eta$ erkennt man $p^{\prime}(z+\omega)>0$ für $0<z<\eta$, so daß in Verbindung mit der aus (4) und (6) folgenden Beziehung $p^{\prime}(\omega \pm \eta)^{2}=4 \alpha^{3}-g_{2} \alpha-g_{3}^{\prime}=\beta^{2}$ schließlich $p^{\prime}(\omega+\eta)=\beta$ im Falle $\beta>0$ sowie $p^{\prime}(\omega-\eta)=\beta$ im Falle $\beta<0$ folgt. Mit $\eta_{0}:=\eta \operatorname{sign} \beta$ falls $\beta \neq 0$ und 
$\dot{\eta}_{0}:=0$ sonst gilt gemäß (4) für die für $\xi \xi \in \mathbb{R}$ reellwertige Funktion $v=v(\xi):=$ $p\left(\xi+\eta_{0}+\omega\right)$

$$
v^{\prime}(\xi)^{2} \equiv 4 \grave{v}(\xi)^{3}-g_{2} v(\xi)^{\prime}-g_{3}, \quad v(0)=\dot{\alpha}, v^{\prime}(0)=\beta, '
$$

so daß sich nach Differentiation $\bar{v}^{\prime \prime}(\xi) \equiv 6 v(\xi)^{2}+c, v(0)=\alpha, v^{\prime}(0)=\beta$ ergibt. Nach Anwendung von (5) für $z=x-t+\eta_{0}$ erhält man insgesamt folgenden

Satz: Diè Lōsung $v=v(x-t)$ der Differentialgleichung (1), die der Bedingung $v(0)=\alpha ; v^{\prime}(0)=\beta(\alpha, \beta \in \mathbb{R})$ genügt, ist gegeben durch

$$
\begin{aligned}
& v(\dot{x}-t):=p\left(x-t+\eta_{0}+\omega\right) \\
& \equiv \frac{1}{4}\left(\frac{p^{\prime}\left(x-t+\eta_{0}\right)}{\left.p\left(x-t+\eta_{0}\right)-u_{0}\right)}\right)^{2}-p\left(x-t+\eta_{0}\right)-u_{0}
\end{aligned}
$$

Manuskripteingang: 22. 03. 1988; in revidierter Fassung 10.'10. 198s'-

\section{VERFASSER:}

Prof. Dr. Horst Herold

Fachbereich Mathematik der. Universität

Lahnberge

D.3550 Marburg/Lahn 